\title{
【Transaction】
}

\section{Development of Smart Textiles for Self-Monitoring Blood Glucose by Using Optical Fiber Sensor}

\author{
Shintaro Kurasawa* , Hiroaki Ishizawa*2, Keisaku Fujimoto*3,\#, \\ Shun Chino*1, and Shouhei Koyama*4 \\ ${ }^{* 1}$ Interdisciplinary Graduate School of Science and Technology, Shinshu University, 3-15-1 Tokida, \\ Ueda City, Nagano 386-8567, Japan \\ ${ }^{* 2}$ Institute for Fiber Engineering, Shinshu University, 3-15-1 Tokida, Ueda City, Nagano 386-8567, Japan \\ ${ }^{*}$ Department of Clinical Laboratory Sciences, Shinshu University School of Health Sciences, 3-1-1 Asahi, Matsumoto City, \\ Nagano 390-8621, Japan \\ ${ }^{* 4}$ Faculty of Textile Science and Technology, Shinshu University, 3-15-1 Tokida, Ueda, Nagano 386-8567, Japan
}

\begin{abstract}
Uninterrupted vital-sign monitoring is one of the application field of the so-called "smart textiles" from the increasing demand for health care demands. Most of the so-called smart textiles have been employed conductive fibers for sensing and signal transformation, but very few employed fiber Bragg grating (FBG) sensor, which can be used as "multi vital sign sensor". Although the demands for blood glucose monitoring is increasing, no smart textile has been investigated from the viewpoint. We demonstrated at the first time in this paper that FBG sensor could sense blood glucose level and could be expected manner in the textile form. We installed the FBG sensor on the subject's wrist and measured pulse wave. The subject had a meal as a glucose load during the measurement. As pulse wave pattern data from FBG sensor system includes information about several other vital signs, we isolated blood glucose information by using hierarchical neural network analysis. The isolated data provided a calibration curve with an extremely high correlation coefficient to the blood glucose. The calculated values are evaluated by dedicated indicator, and we found there are only small error which we can ignore clinically. Comparing with the former studies, we efficiently achieved feature extraction, and the stability of measurement was improved. Although the result represents the first successful method for realizing more accurate non-invasive blood glucose measurement systems aim to smart textile, this result is good enough even at this stage, and we are expecting for practical use in near future. The successful results lead the development of smart textiles for the uninterrupted non-invasive measurement.
\end{abstract}

(Received 7 October, 2019; Accepted 18 December, 2019)

\section{Introduction}

Rise in health awareness and development of various micro-electronic devices including conductive fibers stimulated the trend of smart textiles. Smart textile is suit for monitoring vital signs by featuring long term wearing like normal clothes, however, most of current products can only measure pulse rate or amount of exercises. On the other hands, optical fiber sensors are highly developing recent years, especially, fiber Bragg grating (FBG) sensor has drawn a lot of attention, as it can detect various vital signs with high accuracy. FBG sensor is a sensor that has been proved capable to detect pulse rate, breathing rate, blood pressure and stress loading [1]. In addition, taking advantages of light weight and fiber-shape, it was reported that FBG sensor was woven into textile to make a smart textile [2]. Besides the vital signs which we stated before, the demands for measurement blood glucose is increasing. As the mainstream of current blood glucose measurement instruments are invasive types, a number of problems are invoked in their operation. For instance, the action of pricking a needle causes patient unnecessary stress and poses the risk of blood-borne infection. In addition, replacement of disposable needles makes their operation costly, thus, a need is created for rapid, painless, and risk-free self-blood glucose measuring

\# corresponding author: Keisaku Fujimoto (E-mail: keisaku@shinshu-u.ac.jp; +8 126337 2393) 
methods [3-5]. Moreover, refined sugar is often used in current food circumstances, rapid change of blood glucose can be happened easily than the past. Measurement should be conducted more frequently, rather than in current devices. In light of the drawbacks of current situation, smart textile-based blood glucose measurement provides two big advantages, being non-invasive and longtime monitoring. This paper repots the trial of blood glucose measurement using FBG sensor, in order to give the blood glucose measurement function to smart textile. This FBG sensor is used to obtain pulse wave pattern data characterizing arterial blood flow, which can be affected by glucose concentration. Our technique takes advantage of the high sensitivity and rapid response of FBG sensors, with these characteristics, it is possible to measure several vital signs, including blood glucose levels, fully noninvasively in a few seconds, simply by attaching the optical fiber to the subject's wrist.

In a previous study, we identified the correspondence between a pulse wave measured using the FBG sensor system and a reference blood glucose level using partial least squares regression (PLSR) [6]. Later studies also reported on the relationship between a pulse waveform and arteriosclerosis [7], and between a pulse waveform and diabetes [8]. While these studies show that the correspondence between the different metrics is significant enough in pulse wave measurement as an effective vital sign monitoring, they focused on simple diagnosis of arteriosclerosis or diabetes, and not quantitative estimation of a specific value. Hence, for more concrete measurement of blood glucose, further information regarding its exact relationship to pulse wave data is required. In recent years, machine learning has been used in a variety of medical applications, such as diagnosis of retinopathy from retinal images [9], and diagnosis assistance for Alzheimer's disease [10]. An artificial neural network has also been applied for construction of a calibration curve in blood glucose measurement [11]. Based on these successes, we employ machine learning to relate complex pulse wave pattern data to blood glucose levels, reinforcing the correspondence between pulse waveforms and blood glucose which we reported in a previous paper.

\section{Materials and Methods}

\subsection{Measuring instruments}

The non-invasive measurement system developed in this study requires the construction of a calibration curve. For this, in addition to signals from the FBG sensor, reference values need to be obtained from current blood glucose measurement instruments. In this study, reference measurements were collected using either the Antsense III (HORIBA Co., Ltd., Kyoto, Japan) or the FreeStyle Precision Exceed $\mathrm{H}$ (Abbott Japan Co., Ltd., Osaka, Japan) instruments. The relative uncertainty in invasively collected reference measurements is $3.3-6.5 \%$, when the glucose concentration is in the range of $90-220 \mathrm{mg} / \mathrm{dL}$ [6].

Pulse waves were measured using the PF 25-S 01 FBG sensor system (Nagano Keiki Inc., Japan) [12], configured according to the specification summarized in Table 1. A description of the experimental setup used in measurement is as follows. The system consists of two sensors (FBG Sensor 0 and FBG Sensor 1) connected in series. FBG Sensor 1 has a Bragg wavelength of $1550 \pm 0.5 \mathrm{~nm}$ and is used for detecting pulse wave signals from a subject, based on optical phase shifts (in this sense it can be considered

Table 1 Specifications for the FBG sensor system.

\begin{tabular}{ccc}
\hline \hline \multirow{2}{*}{ Light Source } & Type & Amplified Spontaneous Emission \\
& Power & $30 \mathrm{~mW}$ \\
& Wavelength range & $1525-1575 \mathrm{~nm}$ \\
\hline \multirow{2}{*}{ FBG Sensor } & Length & $10 \mathrm{~mm}$ \\
& Bragg wavelength & $1550 \pm 0.5 \mathrm{~nm}$ \\
& Wavelength resolution & $0.1 \mathrm{pm}$ \\
Detector & Strain resolution & $0.08 \mu \mathrm{m}$ \\
\hline \hline
\end{tabular}


to be the primary sensor). FBG Sensor 0, placed in the chassis end of the device, has a Bragg wavelength of $1530 \mathrm{~nm}$ and is used for correcting phase shifts due to fluctuations in room temperature. Light in the 1525$1575 \mathrm{~nm}$ range is coupled to an optical fiber and transmitted through the optical circulator to FBG Sensor 1, to reflect light at its Bragg wavelength. Reflected light is subsequently transmitted through a Mach-Zehnder interferometer, with the optical path difference set at $3.3 \mathrm{~mm}$. A beam splitter divides this light into three phases, each separated by $2 \pi / 3$ radians, which are then detected using wavelength division multiplexing. Three pairs of detectors record the phase shifts for FBG Sensors 0 and 1. The phase resolution of the system depends on its sampling frequency, which is $10 \mathrm{kHz}$ in this study. Hence, the wavelength resolution of this measuring system is $0.1 \mathrm{pm}[6]$.

\subsection{Pulse wave measurement}

In this experiment, 60 measurements were obtained for each subject. Four healthy males in their twenties were selected for participation. While measurement for each subject was completed at an arbitrary time of day over several hours, we began each measurement from a subject's hungry state, prior to them having a meal. To measure a subject's pulse wave, the FBG sensor was attached to their skin, at a point corresponding to the radial artery, using adhesive medical tape. The subjects lay in a supine position, and their wrists were kept level with their heart. Fig. 1 depicts a pulse wave measurement, illustrating the typical posture of a subject during this process. Measurement was completed in $\sim 20$ seconds, which is the cycle time required for an automatic sphygmomanometer to obtain a stable measurement. Smoothing was then applied to each signal, at a $1 \mathrm{kHz}$ sampling frequency. Then, each pulse wave was filtered using a bandpass filter with a pass band of 0.5-5 Hz. Next, the amplitudes of the signals were normalized, with the maximum set at 1 and the minimum at 0 . Each peak in the signal was selected before it was subsequently normalized signal with respect to time, such that there were 1000 data points on the horizontal axis of each plot. Finally, each signal was averaged to yield a single representative pulse wave. This procedure is described in Fig. 2.

\subsection{Blood glucose measurement}

Blood glucose measurements were performed at the same time as pulse wave measurements, to obtain reference blood glucose values. These references
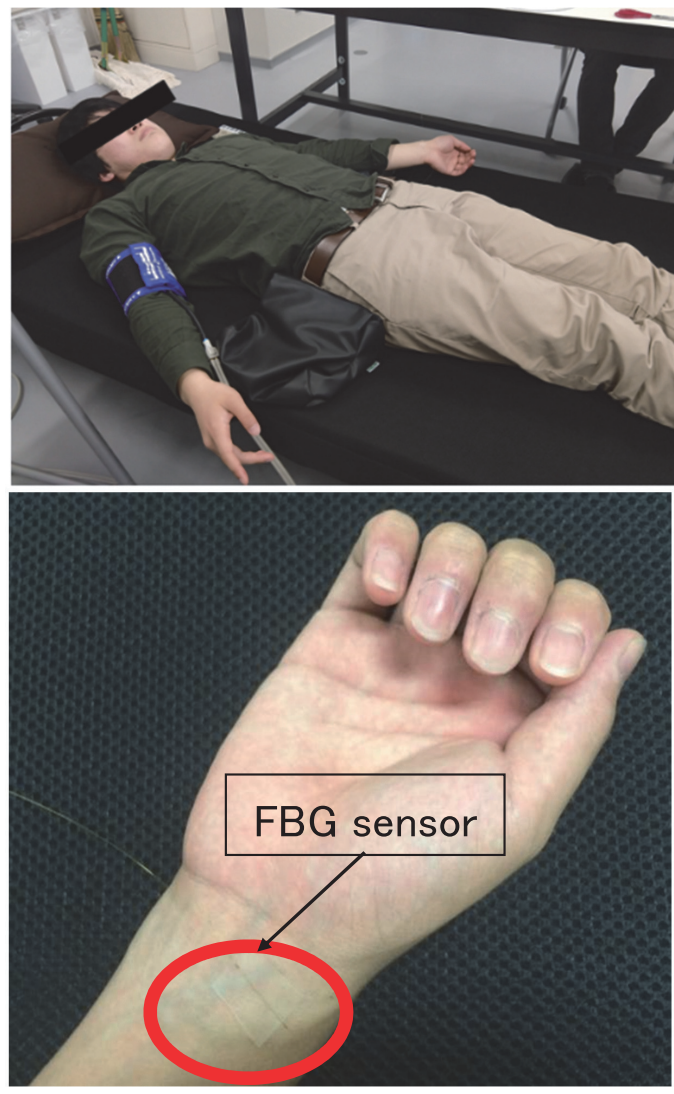

Fig. 1 Representative photograph of a subject during pulse wave measurement and the point to measure.

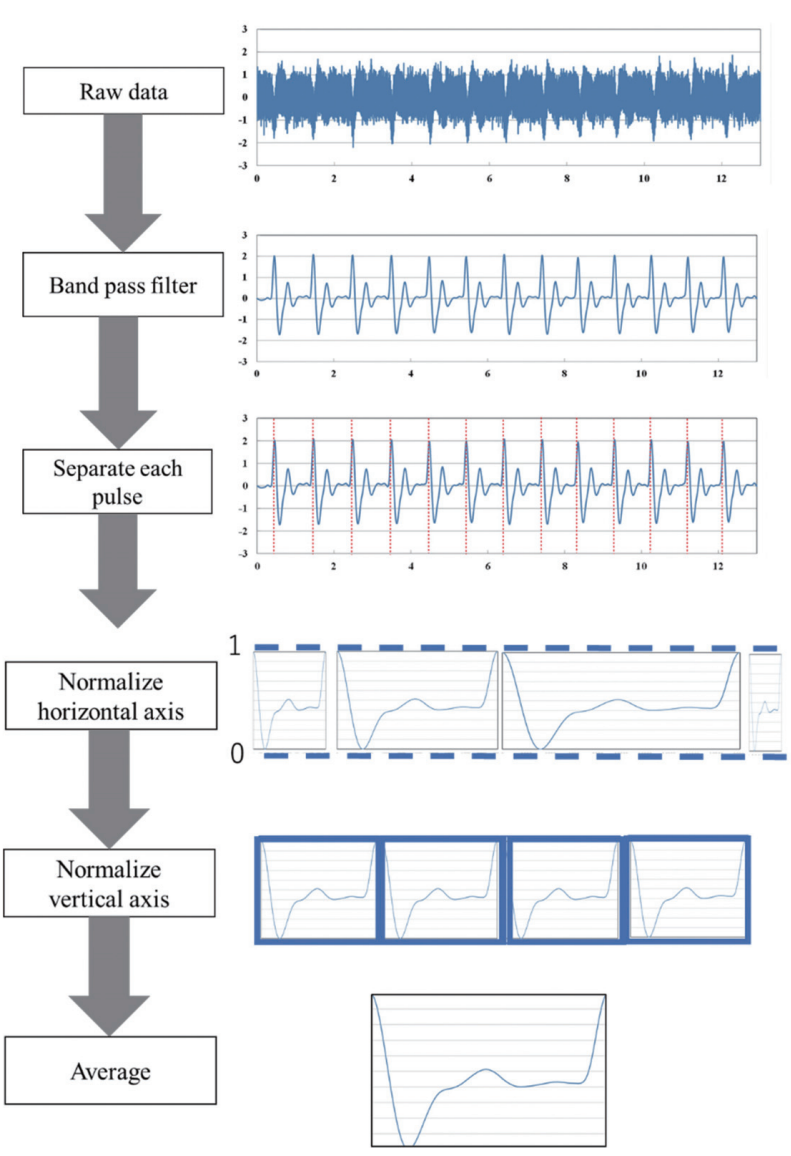

Fig. 2 Schematic flow of pulse wave signal processing. 
were collected using commercially-available invasivetype instruments, such that needle pricking and blood sampling are required. Hence, to ensure our processes met the relevant standard, the protocol for this study was approved by the Ethics Committee of Shinshu University (Project identification code: No. 3202, Verification clinical trial with wearable vital sign measurement system).

\subsection{Machine learning: Hierarchical type neural network (NNW)}

For our machine learning model, the 60 pairs of pulse wave and blood glucose level data were arranged, with pulse wave data defined as the explanatory variable, and blood glucose levels defined as the reference. Subsequently, 50 pairs of data were randomly selected, for constructing the calibration curve. The remaining 10 pairs of data were used to validate the calibration curve. A schematic of the flow of the complete experiment is depicted in Fig. 3.

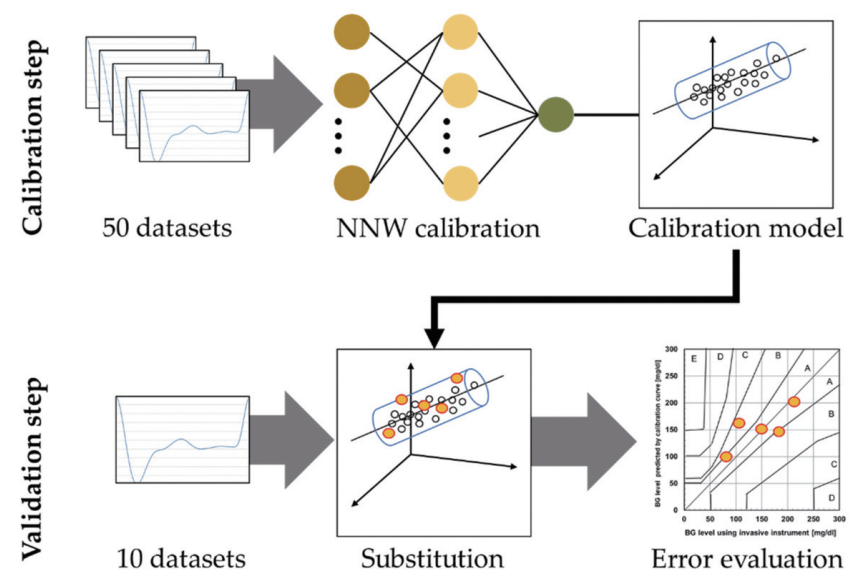

Fig. 3 Schematic flow of the calibration and validation process.

We created our machine learning model using a hierarchical NNW from the MATLAB toolbox, adopting an error propagation method as the learning technique. In addition to the steepest descent method, which is a basic weight update method, we included a weight update method with a moment term, and one that adaptively modifies the learning rate, as fast convergence algorithms. The model featured a threelayer structure consisting of an input layer, an intermediate layer, and an output layer. Learning parameters for the model, adopted from previous research, are summarized as follows. The learning rate was set to 0.1 , the learning rate reduction coefficient was set to 0.9 , the learning rate increase coefficient was 1.03, and the momentum coefficient was 0.85 . The number of middle layer units was 1000 and the number of output layer units was 1 . The learning termination condition occurred either when the learning limit was reached, or a mean squared error of $10^{-4}$ was achieved.

\subsection{Error Grid Analysis (EGA)}

Error grid analysis was used for validating the blood glucose levels estimated using our measurement method. The technique was developed by Parkes et al. [13] to verify the clinical efficacy of blood glucose sensors, and operates as follows. A scatter diagram is constructed, with the reference blood glucose level on the horizontal axis, and the blood glucose level estimated using the measurement method under development on the vertical axis. This diagram is divided into five zones, labeled A-E, defined by the gradient (or the angle between reference and estimated blood glucose levels). A perfect diagonal (i.e. a $45^{\circ}$ angle with no offset) demonstrates a coincidence between the reference and predicted values. Data points with larger gradients than this diagonal represent overestimated results, while points with smaller gradients than the diagonal are underestimated results. EGA determines that a method is clinically acceptable if all results are in zones A and B. In this study, the goal is that all validation results are in the $\mathrm{A}$ or $\mathrm{B}$ zone.

\subsection{Standard Error}

For additional evaluation of our technique, we calculated the standard errors for both the calibration model, and the validation results. The standard error of calibration (SEC) characterizes the error between the reference glucose level and the estimated glucose level used in constructing the calibration curve. Similarly, the standard error of prediction (SEP) characterizes the error between the reference value and the estimate used to validate the calibration curve. These parameters are calculated as below:

$$
\begin{aligned}
& \mathrm{SEC}=\sqrt{\frac{\sum\left(d_{i}-\bar{d}^{2}\right.}{\mathrm{n}-\mathrm{k}}} \\
& \mathrm{SEP}=\sqrt{\frac{\sum\left(d_{i}-\bar{d}^{2}\right.}{n}}
\end{aligned}
$$

where $d_{i}$ is the predicted value, $\bar{d}$ is the average of reference values, $n$ is the number of reference materials, and $k$ is the number of unknown factors.

\section{Results and Discussion}

\subsection{Blood glucose levels}

As blood glucose levels for each subject change 
in response to their activity, such as having a meal or resting, to be suitable for blood glucose calibration, reference values should also vary in a suitably wide range. We obtained the following reference blood glucose levels for each subject, using currently available measurement instruments. Subject A: 81178 mg/dl, Subject B: $93-232$ mg/dl, Subject C: 88-168 $\mathrm{mg} / \mathrm{dl}$, Subject D: $83-207 \mathrm{mg} / \mathrm{dl}$.

\subsection{Pulse waves}

Fig. 4 (a) shows a raw signal obtained from the FBG sensor system during measurement of Subject B's blood glucose at a concentration of $135 \mathrm{mg} / \mathrm{dl}$. This image highlights the need for the signal processing procedures detailed in Section 2.2, as the characteristic pulse waveform is obscured. Fig. 4 (b) shows typical pulse waves obtained from Subject B, corresponding to the maximum, minimum, and mean blood glucose levels recorded, following filtering. These signals represent the average of all pulses obtained in a single measurement, demonstrating that each pulse wave looks similar regardless of the blood glucose level. As it is difficult to distinguish them just by sight, mathematical analysis methods are required. Due to the differing lengths of the time-axis, noise is encountered at the end of each signal. This difference in length is caused by a physiological phenomenon called respiratory sinus arrhythmia, which cannot be removed. Hence, prior to mathematical analysis, the noisy sections were cut, and each signal was normalized with respect to both the horizontal and vertical axes, as shown in Fig. 4 (c).

Calibration curves were constructed for the four different subjects using the pulse waves described in the preceding section. Fig. 5 shows calibration and validation results for blood glucose measurement. Plots on the left are calibration models for each subject, based on NNW analysis. While plots on the right were used in EGA of the 10 datasets obtained from these subjects, for validation of the calibration curves. Table 2 lists results of calibration and validation. The correlation coefficients for all the subjects were over 0.9. Similarly, the SEC values were small, indicating that calibration was completed to a high degree of accuracy. While some deviation could be observed between each subject's SEP, EGA demonstrated that results occupied only the $\mathrm{A}$ and $\mathrm{B}$ zones of these plots, indicating that the error in the system is small enough to be ignored in practical medical situations. These validation results are clinically acceptable [13], suggesting that the proposed

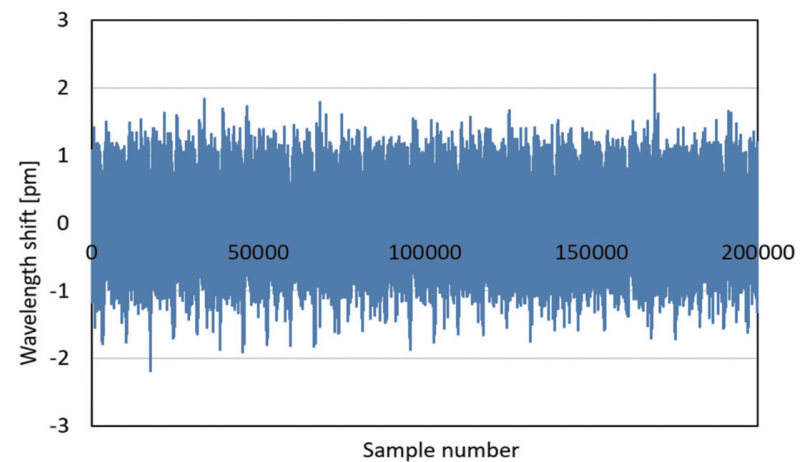

(a)

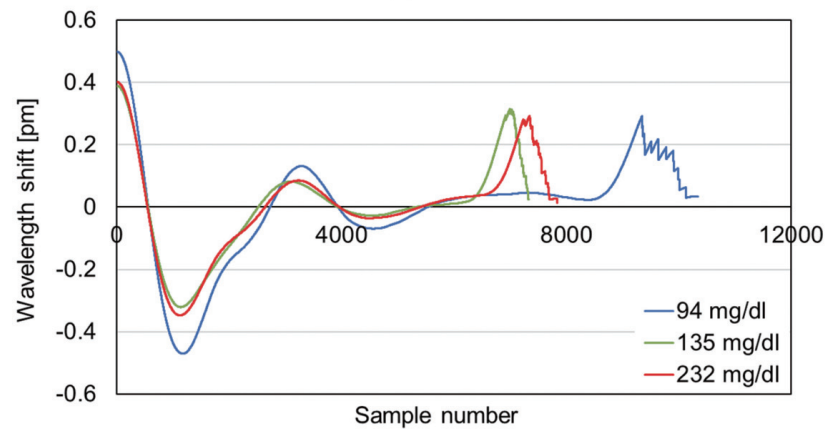

(b)

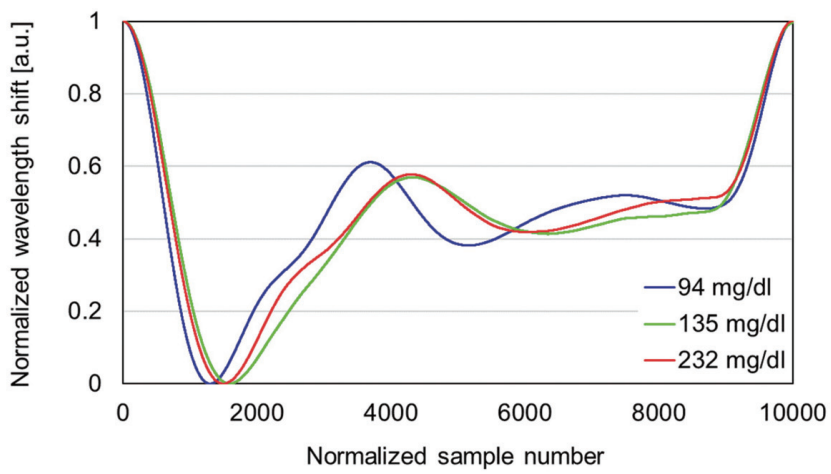

(c)

Fig. 4 Pulse waves recorded from Subject B with their blood glucose levels at their minimum, mean, and maximum values. (a) Raw signal measured at a concentration of $135 \mathrm{mg} / \mathrm{dl}$. (b) Average of all pulse waves obtained in a single measurement (c) Normalized pulse waves.

method is able to measure blood glucose levels adequately.

We obtained high correlation coefficients from all subjects' calibration curves, and a maximum SEC of 10 , which is often considered to be small with regard to blood glucose instruments. This finding indicates that not only do signals from radial arteries obtained by FBG sensing contain information about the blood glucose level, NNW models are able to extract meaningful information from these signals.

We also note that all the validation points are plotted in the A or B zones, suggesting that the system we are proposing is capable of highly accurate 


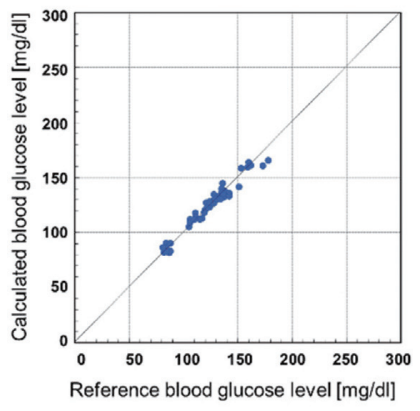

(a)

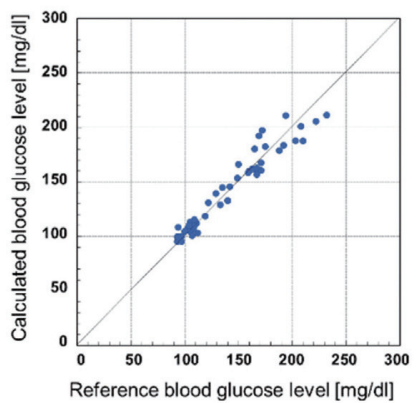

(c)

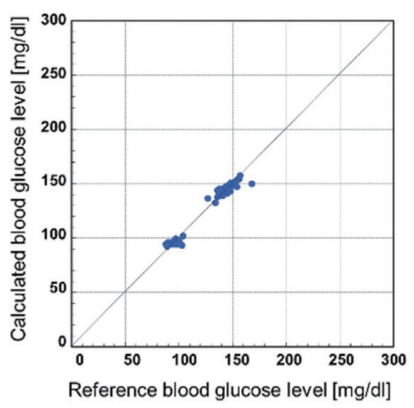

(e)

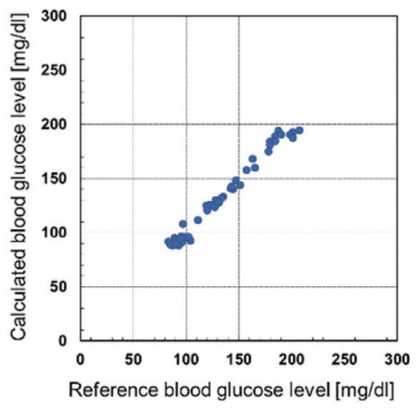

(g)

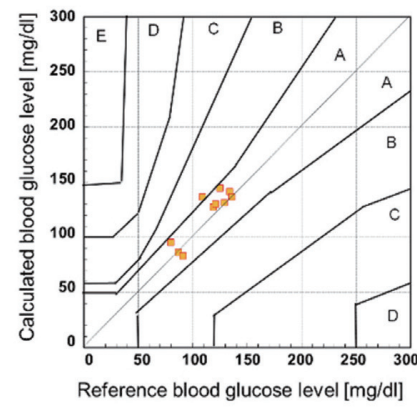

(b)

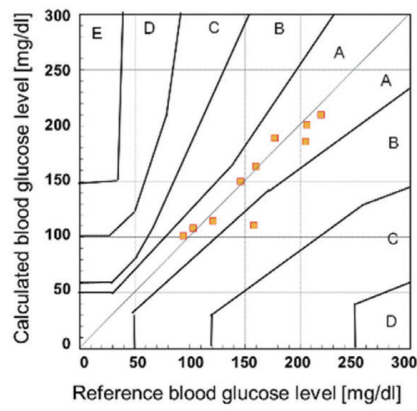

(d)

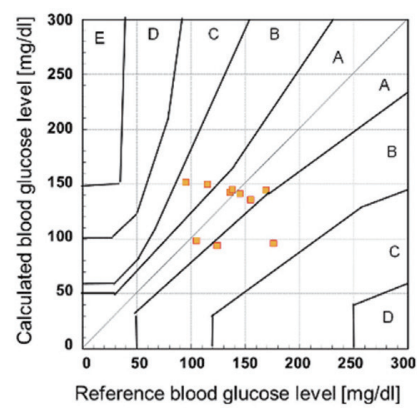

(f)

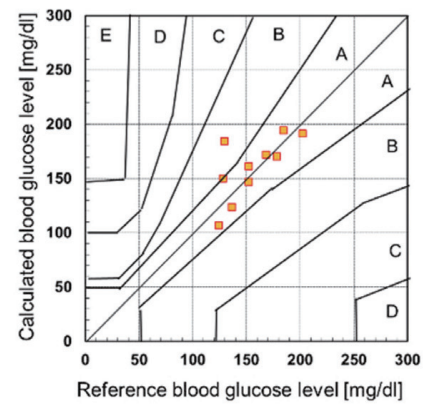

(h)

Fig. 5 Left: Calibration curves for (a) Subject A, (c) Subject B, (e) Subject C, and (g) Subject D. Blood glucose concentrations in these plots were estimated using a neural network. Right: Validation results for (b) Subject A, (d) Subject B, (f) Subject C, and (h) Subject D. Blood glucose concentrations in these plots were estimated using the corresponding calibration curves.

measurement. However, there were differences between each subject's SEP score, which ranged between 14 and $38 \mathrm{mg} / \mathrm{dl}$. In particular, the SEP scores for Subjects C and D were remarkably high. Focusing on Subject C, who had the biggest SEP, only $60 \%$ of data was in the A zone of the EGA plot, the lowest in this study. For analysis of this phenomenon, the ranges of blood glucose levels obtained in validation are listed below, for comparison with the reference values listed in the results section.

Subject A: $80-136 \mathrm{mg} / \mathrm{dl}$.

Subject B: $94-214 \mathrm{mg} / \mathrm{dl}$.

Subject C: $95-176 \mathrm{mg} / \mathrm{dl}$.

Subject D: $124-202 \mathrm{mg} / \mathrm{dl}$. 
Table 2 Results of the calibration and validation

\begin{tabular}{cccccc}
\hline \multirow{2}{*}{$\begin{array}{c}\text { PLS } \\
\text { calibration }\end{array}$} & Subject & A & B & C & D \\
\cline { 2 - 6 } & r & 0.98 & 0.97 & 0.98 & 0.99 \\
& SEC (mg/d) & 5 & 10 & 5 & 5 \\
\hline \multirow{2}{*}{ Validation } & SEP (mg/dl) & 14 & 18 & 38 & 22 \\
& A-zone (\%) & 90 & 90 & 60 & 90 \\
& B-zone (\%) & 10 & 10 & 40 & 10 \\
\hline
\end{tabular}

SEC: standard error of calibration, SEP: standard error of prediction, r: correlation coefficient

From the above, the blood glucose ranges were $56 \mathrm{mg} / \mathrm{dl}, 120 \mathrm{mg} / \mathrm{dl}, 81 \mathrm{mg} / \mathrm{dl}$, and $78 \mathrm{mg} / \mathrm{dl}$ for Subjects A, B, C, and D, respectively. We note that for Subject $\mathrm{C}$, the range of the blood glucose values is about $80 \mathrm{mg} / \mathrm{dl}$ in both calibration and validation. In addition, we also note from Fig. 4 (e) that data points for Subject $\mathrm{C}$ are grouped into two distinct clusters. Further investigation revealed that Subject C's blood glucose level changed drastically because of the glucose load of their regular lunch, causing the unnatural plots observed. Hence, the calibration curve for Subject $\mathrm{C}$ was optimized for narrow and divided datasets, causing the bigger SEP. From this case, we note that a high correlation coefficient is not always reflective of good calibration data, and the technique is reliant on validation data for accurate operation. To address this, data gathering should be repeated with a wider and more continuous range of blood glucose levels.

It should be noted that the validation methods used in this study are only applicable to single datasets. As the shape of a pulse wave depends on the specific patient, generalized calibration and validation can lead to increased errors. We are currently investigating methods to address this problem, as it can affect the validity and certainty of blood glucose measurements. Moreover, the characteristic blood glucose range of healthy subjects and subjects with diabetes differ. While the maximum blood glucose level that can be observed with a healthy subject is typically $\sim 200 \mathrm{mg} / \mathrm{dl}$, this can increase to over $300 \mathrm{mg} / \mathrm{dl}$ or $400 \mathrm{mg} / \mathrm{dl}$ for subjects with diabetes. Hence, the technique in this paper is limited for estimation of healthy blood glucose levels, and further validation is required to extend this to high blood glucose levels. While further study is required before our technique is ready for practical use, the results presented here indicate a direction for how follow up studies should be conducted.

\subsection{Blood glucose measurement using FBG sensors}

We conducted our experiments based on the assumption that continuous pulse wave signals obtained from FBG sensor systems contain information about blood glucose levels, since these signals are similar to the acceleration pulse wave (the second derivative of the fingertip volume pulse wave signal) [12]. Thus, the presence of a correlation between blood glucose level and FBG signals is to be expected, based on the results of the former study. However, in spite of this correlation, there was no visible correspondence between these two parameters, as shown in Fig. 4. Multivariate analysis can make such a correspondence clear [6, 14]. We enumerate some physiological factors suggesting the validity of using pulse wave pattern data for blood glucose sensing below. Which also serve as explanations for why the correspondence between the two parameters is not immediately clear.

The primary reason for measuring blood glucose with FBG sensors was that viscosity is generally considered to change according to glucose concentration. In a former study, it was noted that prediction accuracy improved when the latter part of a pulse wave signal was considered, indicating that the change in blood glucose level caused a change in blood flow with respect to time [6]. Thus, it can be said that blood viscosity or elasticity alters blood flow, leading to a change in the nature of the pulse wave. However, this changing is not directly meaning the changing of viscosity. The most perceptible change in viscosity occurs with high concentrations of glucose that are not typical of the levels observed in human blood. The effect of viscosity is quite small in possible changing blood glucose. In the stage of preexperiment using artificial blood liquid, the difference of viscosity was quite small in range of glucose that 
are typical of the levels observed in human blood. Hence, the correspondence between blood viscosity and blood glucose concentration is more complicated than initial considerations would suggest; at typical human concentrations the effect of viscosity on pulse wave signals is not dominant, and is simply one of several factors that affects blood glucose detection.

To illustrate this, we consider FBG sensor operation, particularly, the effect of anatomical structures on sensor response. Concretely, as FBG sensors are strain sensors, the thickness of structures such as blood vessels, skin, and tissue can affect their response. Different studies have noted the relationship between diabetes and blood vessel stiffness, with elevated blood glucose promoting arteriosclerosis over a long time period. Similarly, over short time periods, high blood glucose level states are also able to change the osmotic pressure of blood plasma. Other reports have stated that both short- and long-term exposure to "High Glucose" and "High mannitol" states decreased the expression of the active, phosphorylated form of endothelial nitric oxide synthase (Ser 1146-eNOS), and, in parallel, increased the expression of the vascular cell adhesion molecule (VCAM)-1 protein [15]. The presence of glucose also affects cell generation and metabolism, as has been detailed elsewhere previously. These phenomena demonstrate how strain - based measurement is valid for sensing blood glucose, even when changes in viscosity are not dominant.

Finally, research conducted by $\mathrm{Li}$ et al. has demonstrated significant differences between the shapes of pulse waves obtained from healthy subjects and subjects with diabetes, in both the amplitude of the wave, and with respect to time. In this study, changes in the arterial pulse waveform parameters of patients with type 2 diabetes were quantitatively assessed. These changes are in addition to the delay of blood flow that has been used as an indicator for arteriosclerosis. Consequently, they were able to conclude that the pulse waveform characteristics could be used as "indices of arterial stiffness" in patients with type 2 diabetes [7]. Similarly, in studying the relationship between pulse wave shape and diabetes, Hao et al. were able to demonstrate a convenient noninvasive pulse-taking diagnostic technique [8].

These studies all indicate a variety of ways in which blood glucose level can affect strain, highlighting the validity of using FBG sensors for measurement. Now, the factor to change the pulse wave form is not only ingredient of blood, but also the characteristic of blood vessel should be considered. However, as the combination of these effects is complex, a suitable classification method is required to obtain accurate blood glucose data, explaining our use of machine learning techniques.

\section{Conclusion and prospects}

In this paper, in order to give the blood glucose measurement function to the smart textile, we have demonstrated a new non-invasive method for measuring blood glucose. Our technique consists of data gathering using an FBG sensor system, construction of a calibration curve using NNW analysis, and subsequent estimation of the blood glucose level. Data calibration and validation was conducted with four subjects, and errors were evaluated using EGA, designed specifically for blood glucose instruments. All validation data points were in the "safe zone" of the EGA plot, indicating that the FBG sensor system and analysis method are suitable for non-invasive blood glucose measurement.

However, we note a limitation in this study, in that all subjects were healthy young males. From previous research it has been noted that the characteristic blood glucose range of healthy subjects and subjects with diabetes differ. To ensure that this technique is valid in all conditions, experiments should be conducted with the cooperation of subjects with diabetes, and with a wider demographic, as supervised by an accredited institution. In addition, the effects of prolonged high-blood glucose levels should be simulated. Hence, this report only details the first steps in the development of the technique.

Nevertheless, the success of our technique suggests that the creation of blood glucose monitoring smart textile is viable. The sensor's fibrous shape makes it suitable for inclusion in a smart textile or garment, particularly as we have already developed some surface treatment techniques that enable the weaving of these devices. Incorporation of these sensor and techniques into textiles would lead to the creation of a revolutionary product: a "wearable multiple vital sign measurement smart textile." The combination of the demand for continuous blood glucose monitoring, these surface treatment techniques, and our promising FBG sensor findings suggest that the establishment of such multi vital sign 
monitoring smart textile is not far in the future.

Author Contributions: Hiroaki Ishizawa, Keisaku Fujimoto and Shouhei Koyama conceived and designed the experiments; Shintaro Kurasawa and Kyoko Katayama performed the experiments; Shintaro Kurasawa and Kyoko Katayama analyzed the data; and Shintaro Kurasawa wrote the paper.

Funding: This research is supported by the "Creation of a development platform for implantable/wearable medical devices by a novel physiological data integration system" project of the Program on Open Innovation Platform with Enterprises, Research Institute and Academia (OPERA) from the Japan Science and Technology Agency (JST). Grant Number JPMJOP 1722.

This work was supported by JSPS KAKENHI Grant Number JP 16 H 01805 and the Wearable vital signs measurement system development project at Shinshu University.

Acknowledgments: This work was supported by a Grant-in-Aid for the Shinshu University Advanced Leading Graduate Program by the Ministry of Education, Culture, Sports, Science and Technology (MEXT), Japan.

Mr. Komatsu of the Seiko Epson Corporation contributed to analysis and experiments when he was a student.

Conflicts of Interest: The authors declare no conflict of interest.

\section{References}

1. S. Koyama, H. Ishizawa, S. Hosoya, T. Kawamura, and S. Chino, Journal of Fiber Science and Technology, 73, 11, 276 (2017).
2. S. Akio, K. Miho, I. Hiroaki, K. Hirokazu, and K. Shouhei, Journal of Textile Engineering, 62, 6, 129 (2016).

3. M. R. Robinson et al., Clinical Chemistry, 38, 9, 1618, (1992).

4. C. E. Ferrante do Amaral and B. Wolf, Med Eng Phys, vol. 30, 5, 541 (2008).

5. N. S. Oliver, C. Toumazou, A. E. G. Cass, and D. G. Johnston, Diabet. Med., 26, 3, 197 (2009).

6. S. Kurasawa, S. Koyama, H. Ishizawa, K. Fujimoto, and S. Chino, Sensors (Basel), 17, 12 (2017).

7 B. Ma, G. Dui, S. Yang, and L. Xin, $C M C$ : Computers, Materials \& Continua, 109, 6, 537 (2015).

8 Y. Hao et al., JMIR Mhealth Uhealth, 7, 4 (2019).

9 D. J. Hemanth, J. Anitha, L. H. Son, and M. Mittal, Journal of Medical Systems, 42, 1 (2018).

10 F. Zhang, Z. Li, B. Zhang, H. Du, B. Wang, and X. Zhang, Neurocomputing, 361, 185 (2019).

11 M. B. Savage, S. Kun, H. Harjunmaa, and R. A. Peura, 29 (2000).

12 Y. Katsuragawa and H. Ishizawa, IEEE International Instrumentation and Measurement Technology Conference (I2MTC) Proceedings, 511 (2015)

13 J. L. Parkes, S. L. Slatin, S. Pardo, and B. H. Ginsberg, Diabetes Care, 23, 8, 1143 (2000).

14 S. Kurasawa, H. Ishizawa, K. Shouhei, K. Fujimoto, and S. Chino, ITMC2017 - International Conference on Intelligent Textiles and Mass Customisation, vol. Session 5 Smart Textiles 3, S253,, (2017).

15. R. Madonna, E. Montebello, G. Lazzerini, M. Zurro, and R. De Caterina, Int J Immunopathol Pharmacol, 23, 3, 755, Sep. (2010). 